\title{
Maximum-likelihood localization of overlapping point sources in 3D microscopy using CLEAN
}

\author{
Michael Handley, Guillem Carles, Andrew R. Harvey \\ School of Physics and Astronomy, Universty of Glasgow, Glasgow, G12 8QQ, UK \\ andy.harvey@glasgow.ac.uk
}

\begin{abstract}
Precise 3D point localization is increasingly important in microscopy, but algorithms break down when PSFs overlap. We adapt the CLEAN algorithm from astronomical imaging to enable MLE localization of high-density datasets. $\odot 2019$ The Author(s)
\end{abstract}

OCIS codes: (180.6900) Three-dimensional microscopy, (100.6890) Three-dimensional image processing, (100.2960) Digital image processing

\section{Introduction}

As the microscopic resolution limit continues to be pushed into the nanoscale, precise localization of fluorescent emitters has become ever necessary. This is especially true of experiments in three dimensions, where the axial position of a particle is inferred from the shape of its defocussed point spread function (PSF). The diffractionlimited nature of the Airy disk restricts the accuracy and depth-of-field of 3D localization, making it a poor choice for this purpose. This can be solved with PSF engineering, by designing a PSF with a large through-focus variation and small accompanying drop-off in intensity. A notable example of this is the double-helix PSF, whose defocusdependent rotation gives improved localization precision in all three dimensions [1].

Localizing an emitter's position from its image requires an appropriate model of the image-forming process and a robust method of estimating the model parameters (i.e. the point's 3D position) which give rise to such measurement. For this purpose, maximum-likelihood estimation (MLE) has been shown to approach the theoretical Cramér-Rao lower bound limit of localization precision [2]. Practically, MLE is an optimization algorithm which maximizes the log-likelihood between the measured PSF and modelled one over the model parameters (xyz position, intensity and background noise). In this regime, the highest log-likelihood gives the best statistical estimate of a particle's position. Phase retrieval MLE [3] extends this capability to work with experimental data for which an analytically modelled PSF might not characterize the aberrations present in practice. Instead, an empirical PSF model is derived from the experimental pupil function, which itself is calculated by phase retrieval of a calibration stack of PSFs.

For it to work efficiently, MLE requires some initial guess of the particle's position, otherwise the process becomes a global optimization over every image coordinate, which can take an inordinate amount of time. MLE also suffers in accuracy in situations of PSF overlap - where two emitters appear close enough together that a single estimation is obfuscated by the presence of the other. Therefore, if MLE is to be used in an algorithm for processing experimental point localization data, it must incorporate an initial estimation of the points' positions and recognize where PSFs overlap and respond appropriately.

\section{MLE-CLEAN}

We have developed an MLE-based localization algorithm which solves the problems of initial estimation and PSF overlap. Our approach derives from CLEAN [4]: an iterative deconvolution algorithm which was originally developed to process radio astronomy observations. In its earliest conception, CLEAN works to identify stars as the brightest points in the image and iteratively subtracts the telescope's PSF centred on each. From this emerges a set of localized points which expands as the brightest PSFs are "cleaned" from the image, revealing the ones below. We have adapted this technique to deal with localization microscopy data in the following way:

1. An initial estimate of a particle's position is found as the maximum coordinate in the cross-correlation with the image and a phase-retrieved model PSF stack.

2. MLE is used with the initial guess to accurately localize the particle.

3. The estimated parameters returned from MLE are used to generate a "cleaning PSF" using the PSF model localized at the particle's position.

4. The cleaning PSF is subtracted from the image. 

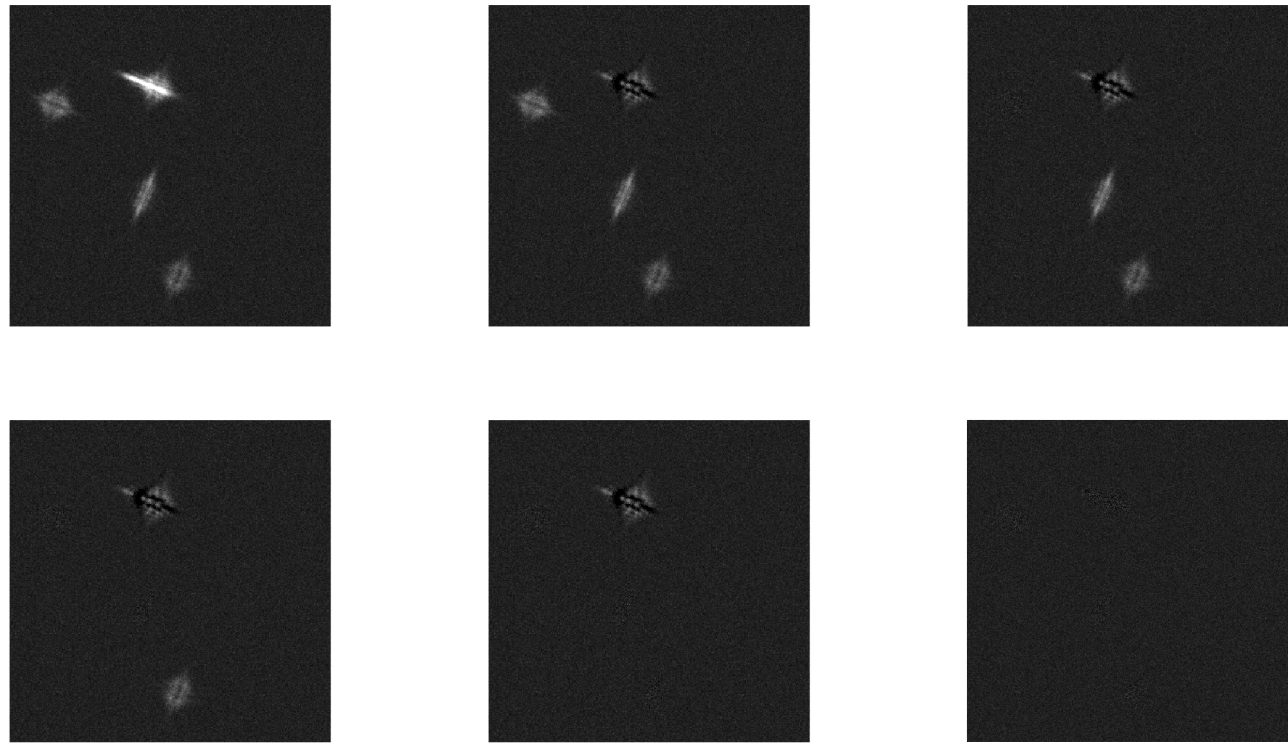

Fig. 1: Steps of the MLE-CLEAN process (top-left to bottom-right) with 5 astigmatic PSFs. Original image (topleft). Fully cleaned image (bottom-right).

The MLE initial estimate problem is solved in the first step of the algorithm by cross-correlation. Since MLE gives an accurate estimation of the PSF's intensity, subtraction of the cleaning PSF from the experimental data leaves only some residual noise. In cases where there is PSF overlap, the algorithm checks after step 1 whether a point has already been localized in the nearby region. If so, the cleaning PSF from that step is added back in and step 2 is performed by maximizing the likelihood of two PSFs instead of one. Each time a new point is found in a region of overlap, the MLE step is run again with an additional PSF. Eventually, the cleaned image approaches the noise floor, at which point all the PSFs in the image should have been found.

Figure 1 gives a visualization of the MLE-CLEAN process for a simulated image of 5 astigmatic PSFs [5]. Two of the PSFs (top-centre of image) overlap. In the first iteration, the algorithm wrongly estimates the overlapping PSFs. In each following iteration, the algorithm correctly estimates the 3D positions of the non-overlapping PSFs and subtracts them from the image. On the final iteration, the first PSF is revisited and correctly estimated as two overlapping PSFs, leaving eventually just noise.

\section{Conclusions}

In conclusion, our algorithm extends the applicability of MLE point localization to high-density situations where PSF overlap would previously impede the technique's accuracy. In combination with a phase-retrieved PSF model, MLE-CLEAN can be applied to any engineered PSF experiment to achieve MLE localization precision and reduce the false positive rate where overlap occurs.

\section{References}

1. S. R. P. Pavani, M. A. Thompson, J. S. Biteen, S. J. Lord, N. Liu, R. J. Twieg, R. Piestun, and W. Moerner, "Three-dimensional, single-molecule fluorescence imaging beyond the diffraction limit by using a doublehelix point spread function," Proceedings of the National Academy of Sciences 106, 2995-2999 (2009).

2. F. Aguet, D. Van De Ville, and M. Unser, "A maximum-likelihood formalism for sub-resolution axial localization of fluorescent nanoparticles," Optics Express 13, 10503-10522 (2005).

3. S. Quirin, S. R. P. Pavani, and R. Piestun, "Optimal 3d single-molecule localization for superresolution microscopy with aberrations and engineered point spread functions," Proceedings of the National Academy of Sciences 109, 675-679 (2012).

4. J. Högbom, "Aperture synthesis with a non-regular distribution of interferometer baselines," Astronomy and Astrophysics Supplement Series 15, 417 (1974).

5. H. P. Kao and A. Verkman, "Tracking of single fluorescent particles in three dimensions: use of cylindrical optics to encode particle position,” Biophysical journal 67, 1291-1300 (1994). 